\title{
Evaluation of convection cooling conditions using Fourier and wavelet analysis in lock-in thermography
}

\author{
by T. Świątczak ${ }^{1}$, R. Olbrycht ${ }^{2}$, B. Więcek ${ }^{3}$
}

Institute of Electronics, Technical University of Łódź, Poland

1tomasz.swiatczak@p.lodz.pl, ${ }^{2}$ robert.olbrycht@p.lodz.pl, ${ }^{3}$ wiecek@p.lodz.pl

\section{Introduction}

Heat dissipation is one of the major problems in modern electronic components. Therefore it is essential to provide proper cooling of such devices, often using forced convection due to its effectiveness. This paper describes the thermal wave method in new application to evaluate convection cooling conditions of electronic devices. In the mentioned method authors investigated the thermal response of the aluminum oxide board with heating resistor. Obtained thermal response was undergone Fourier and wavelet transforms. Given frequency spectrum was analyzed and the authors focused on the phase of thermal response. In addition Fourier and wavelet transforms comparison was performed. Results of the research were presented below.

\section{Thermovision measurements}

\section{Measurement setup and procedure}

A ceramic $\mathrm{Al}_{2} \mathrm{O}_{3}$ substrate with dimensions $50 \times 50 \mathrm{~mm}$ and $0.8 \mathrm{~mm}$ thickness with centrally screen-printed $2 \times 2 \mathrm{~mm}^{2}$ resistor (as shown in Fig.1) has been used to perform research. This resistor was used as a heat source in experiments. The ceramic substrate, not being painted or overglassed, provides high thermal resistance to the ambient, due to the low emissivity of the alumina in contrary to the high emissivity of the resistor's surface. Hence, temperature measurements could be easily carried out by focusing the thermographic camera on the resistor's surface.

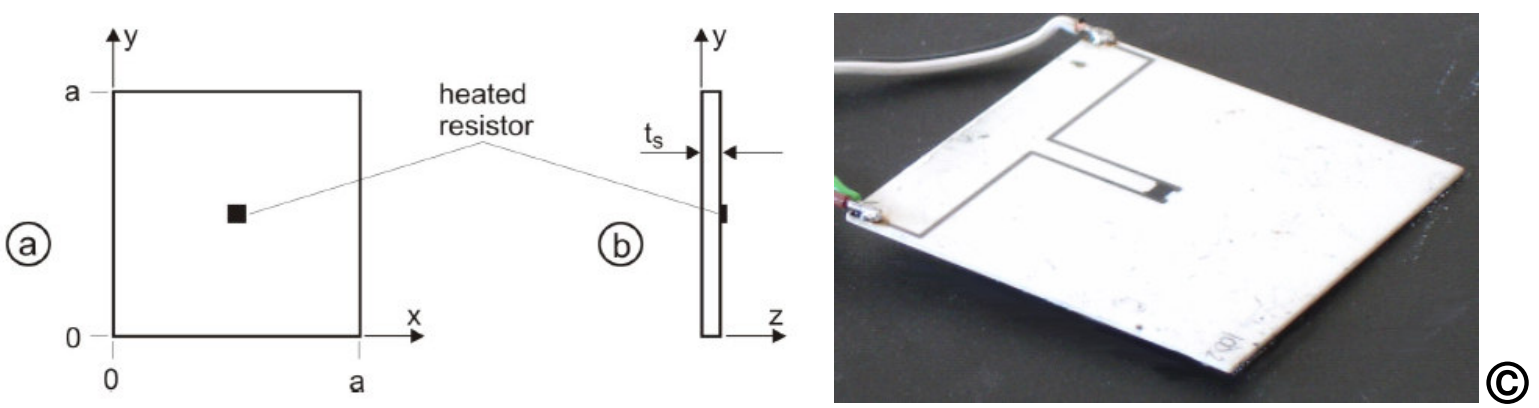

Fig. 1. Front $(A)$ and side $(B)$ view of the substrate shown in (C) [7]

Objects of investigations were placed in wind-tunnel, where with the aid of fan and power inverter, fluent and accurate airflow speed adjustment was possible. The energy was delivered to boards using pulse generator and simple transistor amplifier. The current flew through the resistor placed on aluminum oxide board. Thermal response of the circuit was measured by thermovision camera Inframetrics PM290, computer system for data acquisition and ThermalScope software.

The excitation was a square pulse of the frequency $1 \mathrm{mHz}$. Such a frequency was chosen as a result of boundary element simulation performed in [3] using the electrical equivalent network, and it was found out that the most interesting frequency interval is situated around $1-5 \mathrm{mHz}$. The recorded thermal response for this excitation without forced cooling had a form shown in Fig. 2 and with increasing the airflow speed this response tends to the square signal with lower peak temperatures. 


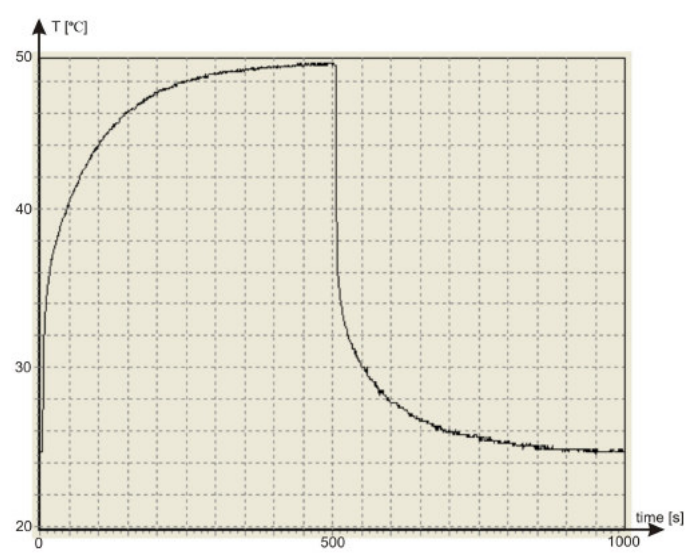

Fig. 2. The thermal response for natural convection

\section{The Fast Fourier Transformation versus the wavelet transform}

In order to convert the time domain into frequency and to obtain the phase values of temperature response FFT was performed. Because previous investigations proved that FFT method is proper tool for this application, authors tried to apply the wavelet transform to check its usability in this field. Complex wavelets were considered, because phase evaluation is possible only from complex results (in this case obtained with the continuous wavelet transform). In particular, phase is calculated using four-quadrant inverse tangent (arctangent) of the each complex result. Results of the wavelet transform calculated with the complex Morlet wavelet are the closest to the FFT results, due to the sinusoidal shape of this wavelet. Hence for comparison of those, the complex Morlet wavelet is a good starting point.

The aim of the research carried by the authors was to obtain monotone characteristics showing the dependence between the airflow speed and the calculated phase. The monotony is an important factor due to the fact that according to the theoretical predictions the dependence should be monotonous. Hence the comparison of FFT and the wavelet transform for this application was done by comparing the monotony of obtained characteristics. Due to the nature of the wavelet transform, phase values are different for different wavelet translations. It is difficult to compare results obtained from FFT and the wavelet transform in terms of exact phase value, unless wavelet parameters are explicitly adjusted in a way that results from both of those transforms are coherent, as in case shown in Fig. 3. Those parameters are discussed below for previously mentioned complex Morlet wavelet, which, according to [6], is defined by equation [1]:

$$
\psi(x)=\frac{1}{\sqrt{\pi f_{b}}} e^{2 i \pi f_{c} x} e^{-\frac{x^{2}}{f_{b}}}
$$

where $f_{b}$ is a constant which defines the width of the wavelet, and $f_{c}$ is the wavelet center frequency constant (which defines the center frequency of the wavelet). When complex Morlet wavelet with $\mathrm{f}_{\mathrm{b}}$ parameter set to infinity and $f_{c}=1$ is used for the wavelet transform, results will be equal to those produced by the FFT (which is then a special case of the wavelet transform). The phase calculation from the wavelet transform is done with fourquadrant inverse tangent (arctangent) of the each complex result.

\section{Results}

As expected, the research revealed that the phase calculation with the wavelet transform using complex Morlet wavelet with $\mathrm{f}_{\mathrm{b}}$ parameter set to infinity and $\mathrm{f}_{\mathrm{c}}=1$, after adjusting the translation of the wavelet as shown in Fig. 3, allowed to obtain practically the same results as with FFT. It proves that FFT may be substituted with the wavelet transform, and both methods are usable for evaluation of convection cooling conditions.

In such a case the recorded thermal response may contain more than one period, but not necessarily an integer number, or worse - there may be only one truncated period recorded. Therefore it is preferred to use a wavelet with windowing function applied in order to take into analysis mainly the middle of recorded sequence, and at the same time higher harmonic number for higher sensitivity.

As far as dynamic range is concerned, one may try to use higher harmonic, as it increases sensitivity of the method. To make the research more complete, this case was also taken into analysis, as well as the situation when recorded thermal response is of unknown parameters, for example from process with internal excitation. 


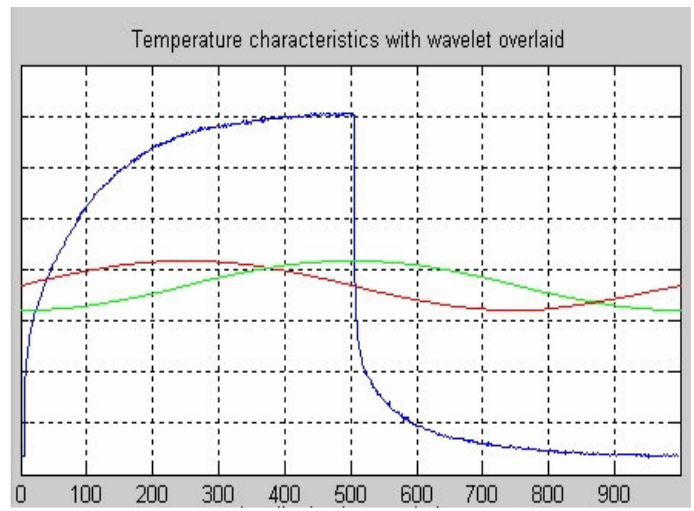

- response to excitation

_ imaginary part of the wavelet

- real part of the wavelet

Fig. 3. Analyzed signal with complex wavelet overlaid, in time domain.

Fig. 4 presents the effect of using $4^{\text {th }}$ harmonic analysis with FFT and wavelet analysis with complex Morlet wavelet. Note that phase plots in this figure were shifted to have common starting point in - 16.6 degrees (this is the starting point of the phase calculated with FFT for $1^{\text {st }}$ harmonic of the signal). As expected, $4^{\text {th }}$ harmonic component is more sensitive than $1^{\text {st }}$ one, unfortunately the monotony is lost in this case. When the wavelet transform is used instead of FFT, due to the Gaussian window which limits applied Morlet wavelet from both sides (as shown in Fig. 5 and 6), there is less sensitivity gain than in case of FFT, but the monotony is preserved. The location and shape of applied complex Morlet wavelet on the background of analyzed signal is shown in Fig. 5. In this case the wavelet transform has advantage over FFT.

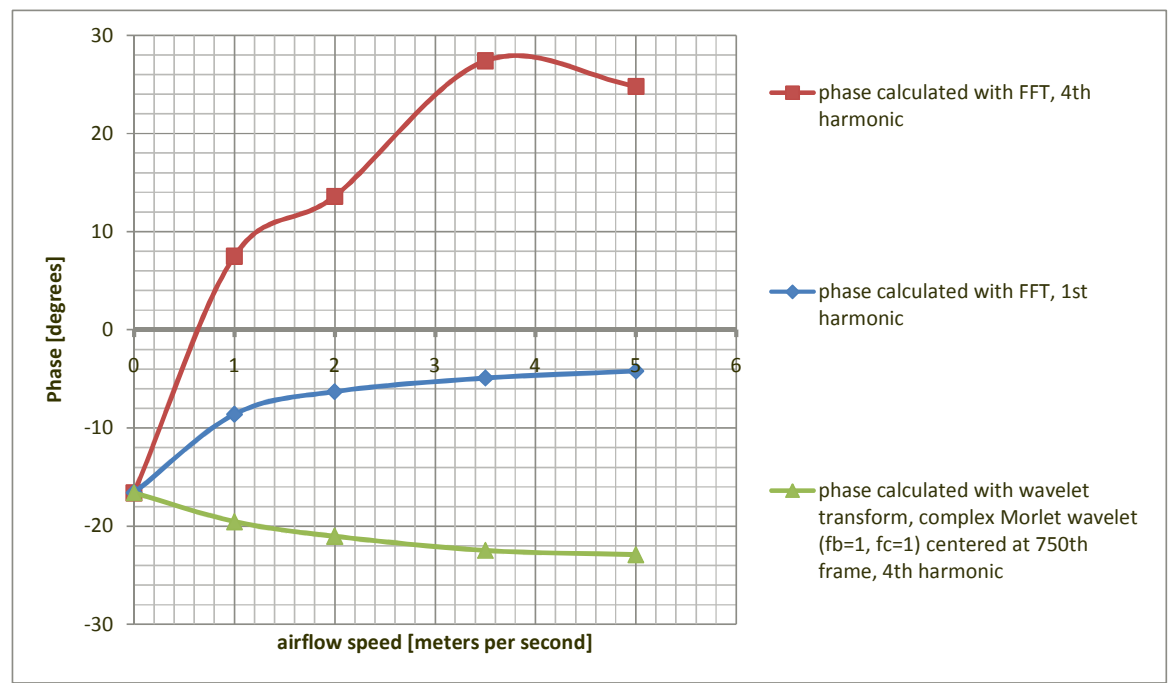

Fig. 4. FFT versus the wavelet transform results for $1^{\text {st }}$ and $4^{\text {th }}$ harmonic

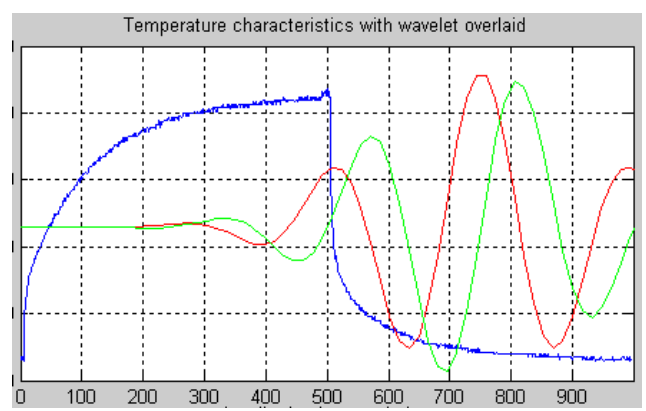

Fig. 5. Signal with complex Morlet wavelet overlaid first (wavelet is centered at $750^{\text {th }}$ frame)

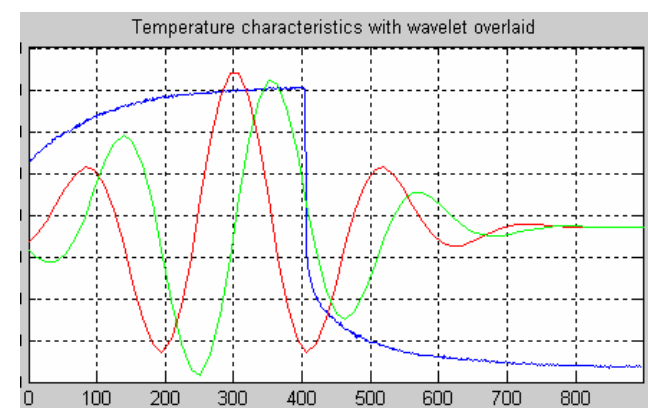

Fig. 6. The same signal as in Fig. 5, but with 100 frames truncated (wavelet is centered at $300^{\text {th }}$ frame)

As mentioned earlier, sometimes the recorded sequence is of unknown parameters, what may happen for example if the thermal excitation is an internal process. Hence one cannot precisely know its fundamental frequency and there is a possibility of truncating the recorded signal. Such a situation was simulated during the research by cutting of first 100 frames of the sequence, as shown in Fig. 6 . The Morlet wavelet was translated to the left, and centered in $300^{\text {th }}$ frame. Such a step provides higher sensitivity compared to the previous case, 
where the wavelet was centered at $750^{\text {th }}$ frame. What is more, the wavelet is not fully damped at $0^{\text {th }}$ frame, what is visible in the form of minor distortion of the calculated phase curve, which is presented in Fig. 7. Again, for better comparison, phase plots were shifted to have common starting point.

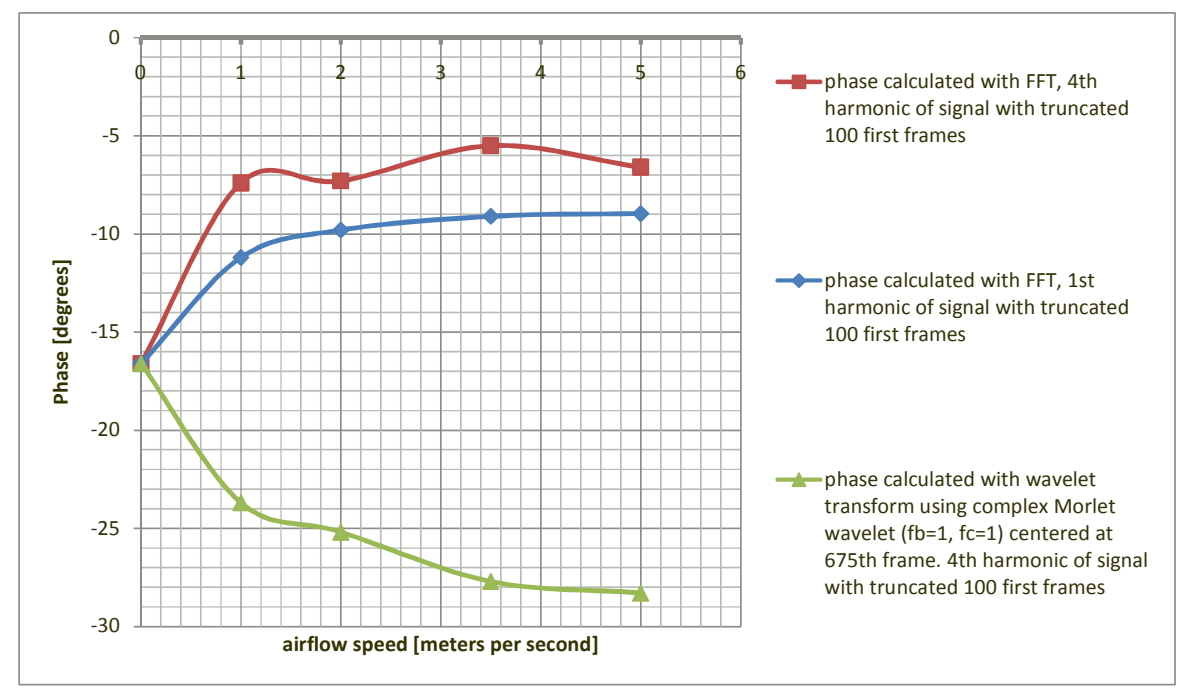

Fig. 7. FFT versus the wavelet transform results for $1^{\text {st }}$ and $4^{\text {th }}$ harmonic of truncated signal

\section{Conclusions}

First of all it was demonstrated that FFT can be substituted with the wavelet transform as far as convection cooling condition estimation is concerned. What is more, adjusting the parameters of the wavelet one gains a powerful tool to find a compromise between the sensitivity of the method and desired monotony of the phase curves.

From Fig. 4 it is clearly visible that for higher harmonic FFT may not guarantee monotone results despite the high dynamics. On the other hand the result obtained with the wavelet transform for the same harmonic number is of much better shape, and resembles the FFT result for $1^{\text {st }}$ harmonic, although with the mirror flip (i.e. ascending curve becomes descending). This proves that the wavelet transform provides results which are less likely to be distorted and non-monotone.

From Fig. 7 it can be observed that adjusting the translation of wavelet provides better sensitivity, but one should be careful - it is easy to introduce distortions to the phase curve for the price of high sensitivity. It is vital to use such wavelet parameters that will make the wavelet muted in the truncated areas of analyzed signal. In other words one must balance the monotony of the phase and sensitivity. As an example, observe the minor distortion of the phase calculated with the wavelet transform, compare it to the much greater distortion obtained with FFT and relate those distortions to the shape of wavelet in Fig. 6.

Further research may also include involving other wavelets for analysis, such as Daubechies. The criteria for selection of appropriate wavelet could be based on matching the shape of the wavelet to the shape of analyzed signal.

\section{REFERENCES}

[1] R. Olbrycht, B. Więcek, G. Gralewicz, T. Świątczak, G. Owczarek „Comparison of Fourier and wavelet analyses for defect detection in lock-in and pulse phase thermography”, QIRT Journal, Vol. 4 - No 2-2007

[2] T.Świątczak, B.Więcek, K.Tomalczyk "Convective cooling evaluation of electronic devices using lock-in thermography", 14 th International Conference, MIXDES 2007, Ciechocinek, ISBN 83-922632-4-3 212306 2007,

[3] T.Świątczak, B.Więcek, K.Tomalczyk "Characterisation of convection cooling for electronic circuits by frequency analysis", ICSES 2006 International Conference on Signals and Electronic Systems, Łódź, 1720092006

[4] R. Olbrycht, "Applications of thermal wave method for material properties investigation", M. Sc. Thesis, Institute of Electronics, Technical University of Lodz, 2007

[5] B. Staniszewski: "Wymiana ciepła" Państwowe Wydawnictwo Naukowe, Warszawa, 1979

[6] A. Teolis, "Computational signal processing with wavelets", Birkhauser, 1998 